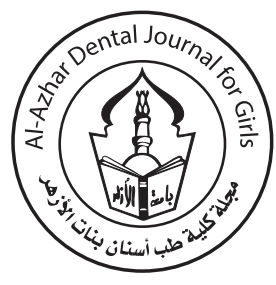

\title{
The Effect of pH Cycling on Surface Microhardness and Fluoride Release of Two Modified Nanoclay Glass Ionomer Restorations In Class V Cavities
}

\author{
Monaliza M. Abdelaziz ${ }^{1 *}$, Maha A. Niazy ${ }^{2}$, Hebaallah M. Taher ${ }^{3}$
}

Codex : 64/20.10

azhardentj@azhar.edu.eg

http://adjg.journals.ekb.eg

DOI: $10.21608 /$ adjg.2020.13538.1163

Restorative Dentistry

(Removable Prosthodontics, Fixed

Prosthodontics, Endodontics, Dental Biomaterials, Operative Dentistry)

\section{KEYWORDS}

Fluoride release,

Glass Ionomer,

Microhardness,

Montmorillonite,

Nanoclay.

\begin{abstract}
Purpose: To assess the effect of $\mathrm{pH}$ cycling on surface microhardness and fluoride release of two modified nanoclay glass ionomer restorations (in vitro). Materials and Methods: A total of 180 permanent human caries free premolars were used. Class V were prepared on facial surfaces of the teeth. They were classified according to type of glass ionomer used for restoration of the cavities into three groups (60 teeth each); conventional glass ionomer medifill (A1), Dellite 43 modified glass ionomer (A2), and Dellite LVF modified glass ionomer (A3). Each group was moreover subdivided into three subgroups (20 teeth each) relative to the immersion solution used. The First subgroup remained in distilled Water (B1 control), the second subgroup was stored in artificial Saliva (B2) whereas the third subgroup was subjected to $\mathrm{PH}$ cycling (B3). Microhardness and fluoride release were measured after 7 and 30 days respectively. Results: There was a significant difference in the mean microhardness and fluoride release between the different materials after $\mathrm{pH}$ cycling $(\mathrm{p} \leq 0.001)$. PH cycling reduced the mean microhardness of the materials but induced the highest fluoride release. Conclusion: The proposed $\mathrm{pH}$ cycling model affected the microhardness of the tested materials while improved their fluoride release. Although the incorporation of montmorillonite into glass ionomer liquid improved the microhardness of the materials but it had yet, no effect on its fluoride release.
\end{abstract}

\section{INTRODUCTION}

Glass ionomer cements (GICs) are interesting dental materials catching the attention clinically and have particular characteristics that make them useful adhesive materials for restoration of carious cavities

- Paper extracted from $\mathrm{PhD}$ thesis titled: Evaluation of pulp reaction to two modified nanoclay glass ionomer restorations and influence of $\mathrm{pH}$ cycling on their microhardness and fluoride release.

1. Assistant lecturer of Conservative Dentistry, Faculty of Dentistry, October 6 University, Cairo, Egypt

2. Professor of Operative Dentistry, Faculty of Dental Medicine for Girls, AL-Azhar University, Cairo, Egypt

3. Assistant Professor of Conservative Dentistry, Faculty of Dentistry, Cairo University, Cairo, Egypt

* Corresponding author email: monalizamaher@gmail.com 
located in low-stress bearing areas ${ }^{(1)}$. These properties include chemical bonding to different tooth tissues, acceptable pulp reaction, low coefficient of thermal expansion, low toxicity and ability to release fluoride (2). Although having a lot of advantages, GICs suffer from some problems such as decreased fracture toughness and increased degree of occlusal wear when comparing it to other restorative materials, such as amalgam and resin composite restorations ${ }^{(3)}$.

Montmorillonite (MMT) is a crystalline structure formed of layers of clay mineral. Each layer of clay consists of alumina octahedral sheet in the centre, between two silica tetrahedral plates through getting over the forces among the layers, small molecules may penetrate and polymerized inside clay architecture. Montmorillonite (MMT) nanoclays became one of the most interesting materials for research through last years. Because of large interface of the polymer-nanoclay reciprocal action, it consider an attractive martial for dispersion relation in polymers ${ }^{(4)}$.

The integration of little quantity of montmorillonite in the polymer matrix much improves the characteristics of the resulting compound when compared to classical one. Through penetration of low weight proportion of nanoclay into polymer matrix, polymer-nanoclay structures are formed ${ }^{(5)}$. The application of many types of nanoclays in restorative materials leads to improvement of mechanical, physical, and adhesive attitudes ${ }^{\left({ }^{6}\right)}$. The mechanical fulfilment (hardness and strength) showed much enhancement particularly after incorporation of little amount of nanoclay into polymers.

One of the most important factors when choosing a restorative material is its mechanical properties. As the restorative material is applied to compensate for the lost tooth structure, it needs to be robust enough to resist the forces associated with mastication. Hardness test is executed to examine these mechanical properties ${ }^{(7)}$.
Fluoride release pattern of GIC achieves a high rate of release at first then a noticeable decrease in the release rate after period of time. Previous invitro studies found that, GIC showed a decreased, prolong-dated and release of fluoride ions at constant rate, which can carry on for many years ${ }^{(8,9)}$.

Resembling the oral cavity condition is mandatory for the method of restorative materials evaluation, especially regards to changes in the $\mathrm{pH}$ of dental plaque. The $\mathrm{pH}$-cycling represent the dynamic balance between demineralization and remineralization which simulates the clinical status. When the materials are compared regards to fluoride release using distilled water and $\mathrm{pH}$-cycling model, variations may become obvious. However, the effect of $\mathrm{pH}$ alteration on the execution of restorative materials cannot be determined well ${ }^{(10)}$.

Accordingly, this study was conducted to clarify the effect of $\mathrm{pH}$ cycling on surface microhardness and fluoride release of two modified nanoclay glass ionomer restorations.

\section{MATERIALS AND METHODS}

Conventional glass ionomer restoration (Medifill ${ }^{\circledR}$ Promedica (dental material) Lot:1719471 Germany. www.promedica.de) was used in the study. Two types of montmorillonite (Dellite ${ }^{\circledR} 43 \mathrm{~B}$ with organic component and Dellite ${ }^{\circledR}$ LVF- Laviosa (advanced mineral solution) Italy. www.laviosa. com) were used as modifiers.

\section{Preparation of nanoclay modified glass ionomer:}

The two types of montmorillonite (MMT) powder were dispersed in medifill liquid by the exfoliation-adsorption method ${ }^{(11)}$. Dellite ${ }^{\circledR} 43 \mathrm{~B}$ and Dellite ${ }^{\circledR}$ LVF MMT weights were measured by a balance $(0.375$ grams $=2.0 \mathrm{wt} . \%)$ and then mixed on a hot tray for 24 hours at $75^{\circ} \mathrm{C}$ with speed of 100 rpm using a magnetic-stirrer.

A total of 180 permanent human premolar teeth free from caries were used. They were classified 
according to type of glass ionomer used for the restoration into three main groups (60 teeth each). Conventional glass ionomer was used (A1) in the first group. In the second group, Dellite 43 modified glass ionomer was used (A2), while in the third group, Dellite LVF modified glass ionomer was used (A3). Each group was moreover subdivided into three subgroups equal to each other relative to the storage solution (20 teeth each). The first subgroup was sunken in distilled water (B1 negative control), the second subgroup was sunken in artificial saliva (B2) and the third one was subjected to PH cycling (B3) through the immersion of teeth in deminerarilizing - remineralizing solutions (CocaCola - artificial saliva) ${ }^{(12)}$. Each subgroup was further subdivided into two classes (10 teeth each) relative to the immersion period 1 Week (C1), 1 Month, (C2) respectively. Variables of the study are showed in table 1 .

Table (1) Variables of Study

\begin{tabular}{|c|c|c|}
\hline Variable & Symbol & Denote \\
\hline \multirow{3}{*}{$\begin{array}{c}\text { Glass } \\
\text { Ionomer (A) }\end{array}$} & $A_{1}$ & Conventional glass ionomer \\
\hline & $\mathrm{A}_{2}$ & Dellite 43 modified glass ionomer \\
\hline & $\mathrm{A}_{3}$ & Dellite LVF modified glass ionomer \\
\hline \multirow{3}{*}{ Solution (B) } & $\mathrm{B}_{1}$ & Distilled Water \\
\hline & $\mathrm{B}_{2}$ & Artificial Saliva \\
\hline & $\mathrm{B}_{3}$ & PH Cycling \\
\hline \multirow{2}{*}{$\begin{array}{l}\text { Immersion } \\
\text { Period (C) }\end{array}$} & $\mathrm{C}_{1}$ & 1 week \\
\hline & $\mathrm{C}_{2}$ & 1 Month \\
\hline
\end{tabular}

Standardized trapezoidal class $\mathrm{V}$ cavities with $2 \mathrm{~mm}$ mesiodiostal width, $2 \mathrm{~mm}$ in the occulso-gingival height and depth of $1.5 \mathrm{~mm}$ were prepared in the cervical one third of the facial surfaces of teeth with the preparation extending $1 \mathrm{~mm}$ above the CEJ. Each tooth was stabilized firstly by vice tool ( PD-374 Hobby Vise (Max opening 40mm /width $60 \mathrm{~mm}$ ), Pro's kit- a mechanical apparatus used to secure an object to allow work to be performed on it) to ensure standardization of the prepared cavi- ties. Cylindrical fissure carbide bur (Komet, 454 South Anderson Road, Suite 14 Rock Hill, SC 29730, USA; \#H21, 204, size 010) revolving at high speed range was used under copious air water spray coolant. Each cavity was measured with a William's graduated probe to uniform size ${ }^{(13)}$.

The prepared cavities were air - water sprayed and then dried with air syringe. The previously mentioned types of glass ionomer were applied in the prepared cavities. According to the manufacture, one scope of powder was mixed with one drop of liquid on glass slab and applied in each cavity by suitable condenser (Double Dental Plugger W381001, Guilin Woodpecker Medical Instrument Co., Ltd.). The excess restorative material was removed using a finishing diamond stone with small grit** at high speed (100,000 rpm). The specimens were kept for 1 hour in an incubator at $37^{\circ} \mathrm{C}$ after restoration to resemble the normal oral environment ${ }^{(14)}$.

Each restorative group of the specimens were sectioned into three subgroups respective to the storage solution. The specimens of the first subgroup were placed in distilled water as a negative control $(\mathrm{PH}=7)$. The specimens of the second subgroup were kept in artificial saliva which was prepared in analytical chemistry department, faculty of pharmacy, Cairo University. It consisted of (1.72gm) hydrous sodium bicarbonate, $(0,468 \mathrm{gm})$ sodium hydrophosphate and $(0.11 \mathrm{gm})$ calcium chloride diluted in 1 liter of distilled water $(\mathrm{PH}=7.3)$ (15). The specimens of the third subgroup undergone $\mathrm{PH}$ cycling through daily immersion of specimens in demineralizg-remineralizing media (Coca-Cola -artificial saliva). Each specimen was immersed 6 hours in Coca-Cola of $(\mathrm{PH}=2,6)$. It consisted of Fluoride (0.13 ppm F), Calcium-(0.84 mM Ca $\left.{ }^{2+}\right)$, titratable acid $(83.6 \mathrm{mmol} / \mathrm{L} \mathrm{OH}$ and $40.0 \mathrm{mmol} / \mathrm{L}$ $\mathrm{OH}$ to $\mathrm{pH} 5.5)$, Phosphate $(5.43 \mathrm{mM} \mathrm{Pi}),{ }^{(12)}$ at $37{ }^{\circ} \mathrm{C}$ then they were rinsed with distilled water then dried with absorptive paper and sunken in artificial saliva for 18 hours. Each sample was stored in $10 \mathrm{ml}$ of the previous studied solutions individually in a graduated plastic container until the two immersion 
periods, 7 and 30 days. The solutions were changed every day and $1 \mathrm{ml}$ of each was gathered for fluoride analysis.

\section{Microhardness assessment:}

The samples were dried with absorbent after their removal from different storage medium at the determined periods. Vickers microhardness tester (Wilson Hardness, 825 University Avenue Norwood, MA, USA; Wilson hardness, tukon 1102\1202) was used for microhardness evaluation. The samples were mounted individually in cylindrical acrylic block so that the exposed surface was set parallel to the horizontal plan, immobilizing the specimen during measurements which were investigated at room temperature. The exposed surface of each tooth was progressively undergone polishing with an automatic polishing machine under flow of water with waterproof silicon carbide grinding paper. These procedures were managed to create a parallel flat surface for the Vickers microhardness tests ${ }^{(16)}$.

Three indentations were obtained by Vickers diamond under $50 \mathrm{gm}$. for load of 10 seconds. The diamond shaped indentations were recorded by an optical microscope with digital camera and analyse the image by special software, allowing proper digital reading of their diagonal. The average lengths of diagonals were used to compute the microhardness values (HV). The ratio $\mathrm{F} / \mathrm{A}$ reported the $\mathrm{HV}$ number, where $\mathrm{F}$ is the force fallen on the diamond in kilograms-force, A is the area of the created indentation square in millimetres square. Where $d$ is the average length in millimetres of the diagonal obtained by the indenter. Hence $\mathrm{HV}=0.1891 \mathrm{~F} / \mathrm{d}^{2}{ }^{(17)}$. So, the higher the average of diagonals lengths, the less microhardness value.

\section{Fluoride release analysis:}

The cumulative fluoride ion density of each solution was quantified after each immersion period using high-pressure ion chromatography (Dionex ICS-5000; Thermo Fisher Scientific, 168 Third Avenue, Waltham, MA USA 02451). The analyte (extracted sample) was filtrated on $0.45 \mu \mathrm{m}$ and Diluted with deionized water. The sample was injected inside the system through an injection valve. The sample loading was done at atmospheric pressure. After converting the injection valve, the sample was transferred to the separator by the mobile phase to interact with the stationary phase medium. This chemical exchange occurs at different levels for each type of ion in the analyte. Quantitative results were collected by investigating the peak area or peak height, both of which are proportional to the concentration of the analyte. Signals were taken by detector connected to a computer with a proper chromatography software.

\section{Statistical Analysis:}

Three-way ANOVA was used to judge between materials, followed by pairwise comparison with Bonferroni correction $(\alpha=0.05)$.

\section{RESULTS}

\section{I- Microhardness}

Mean and standard deviation for microhardness (HV) are showed in table (2) and figure (1). In the first interval, 1 week (C1) the highest mean microhardness was recorded with Dellite 43B modified glass ionomer (A2) when it was immersed in artificial saliva (B2) (36.39 \pm 1$)$, whereas the lowest mean microhardness was recorded with conventional glass ionomer (A1) when it was subjected to $\mathrm{pH}$ cycling (B3) (14.64 \pm 1.89$)$. ANOVA test reported that the difference between groups was statistically significant $(\mathrm{p} \leq 0.001)$. In the second interval, one month (C2), the highest mean microhardness was recorded with Dellite 43B modified glass ionomer (A2) when it was immersed in artificial saliva (B2)( $36.01 \pm 2.45$ ), whereas the lowest mean microhardness was recorded with conventional glass ionomer (A1) when it was subjected to $\mathrm{pH}$ cycling (B3)

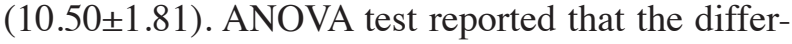
ence between groups was statistically significant $(\mathrm{p} \leq 0.001)$. 
Table (2) Mean and standard deviation \pm for Microhardness (HV) of different immersion media with the other variables.

\begin{tabular}{|c|c|c|c|c|c|c|}
\hline & & & B1 & B2 & B3 & p-value \\
\hline \multirow{6}{*}{ Microhardness (HV) } & \multirow{2}{*}{ A1 } & C1 & $19.12 \pm 2.37^{\mathrm{a}}$ & $18.41 \pm 2.52^{\mathrm{a}}$ & $14.64 \pm 1.89^{b}$ & $\leq 0.001 *$ \\
\hline & & $\mathrm{C2}$ & $19.01 \pm 2.25^{\mathrm{a}}$ & $18.80 \pm 2.07^{\mathrm{a}}$ & $10.50 \pm 1.18^{b}$ & $\leq 0.001 *$ \\
\hline & \multirow{2}{*}{$\mathbf{A} 2$} & C1 & $35.55 \pm 2.73^{\mathrm{a}}$ & $36.39 \pm 1.00^{a}$ & $24.08 \pm 2.24^{b}$ & $\leq 0.001 *$ \\
\hline & & $\mathrm{C2}$ & $34.76 \pm 4.25^{\mathrm{a}}$ & $36.01 \pm 2.45^{\mathrm{a}}$ & $19.59 \pm 1.01^{b}$ & $\leq 0.001 *$ \\
\hline & \multirow{2}{*}{ A3 } & C1 & $27.66 \pm 1.40^{\mathrm{a}}$ & $28.54 \pm 0.73^{\mathrm{a}}$ & $19.31 \pm 1.95^{b}$ & $\leq 0.001 *$ \\
\hline & & $\mathrm{C2}$ & $26.89 \pm 2.69^{a}$ & $28.57 \pm 0.80^{a}$ & $12.57 \pm 1.20^{\mathrm{b}}$ & $\leq 0.001 *$ \\
\hline
\end{tabular}

Different letter within each row indicates significant difference. $\quad *$ =Significant, NS=Non-significant

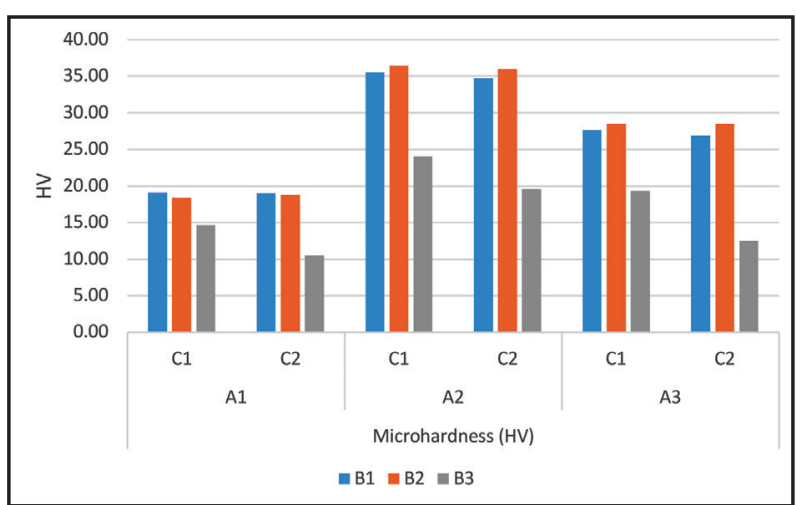

Figure (1) Bar chart showing mean Microhardness (HV) of different immersion media with the other variables.

\section{II- Fluoride release}

Mean and standard deviation for fluoride release are presented in table (3) and figure (2). In the first interval, 1 week $(\mathrm{C} 1)$ the highest mean fluoride re- lease was recorded with Dellite 43B modified glass ionomer (A2) when it was subjected to $\mathrm{pH}$ cycling (B3) $(16.89 \pm 1.62)$, whereas the lowest fluoride release was recorded with conventional glass ionomer (A1) when it was immersed in artificial saliva (B2) $(1.43 \pm 0.43)$. ANOVA test reported that the difference between groups was statistically significant $(p \leq 0.001)$. In the second interval, one month (C2), the highest fluoride release was recorded with Dellite LVF modified glass ionomer (A3) when it was subjected to $\mathrm{pH}$ cycling (B3) (23.46 \pm 0.77$)$, whereas the lowest fluoride release was recorded with Dellite LVF modified glass ionomer (A3) when it was immersed in artificial saliva (B2)( $1.03 \pm .0 .31)$. ANOVA test reported that the difference between groups was statistically significant $(\mathrm{p} \leq 0.001)$.

Table (3) Mean and standard deviation \pm for fluoride analysis ( $\mathrm{mg} / \mathrm{l})$ of different immersion media with the other variables.

\begin{tabular}{|c|c|c|c|c|c|c|}
\hline & & & B1 & B2 & B3 & p-value \\
\hline \multirow{6}{*}{ Fluoride analysis $(\mathrm{mg} / \mathrm{l})$} & \multirow{2}{*}{ A1 } & C1 & $3.31 \pm 0.63^{b}$ & $1.43 \pm 0.43^{\mathrm{c}}$ & $16.22 \pm 1.10^{\mathrm{a}}$ & $\leq 0.001 *$ \\
\hline & & $\mathrm{C2}$ & $3.25 \pm 0.62^{b}$ & $1.05 \pm 0.50^{\mathrm{c}}$ & $22.90 \pm 0.96^{\mathrm{a}}$ & $\leq 0.001 *$ \\
\hline & \multirow{2}{*}{ A2 } & C1 & $2.96 \pm 0.58^{b}$ & $1.53 \pm 0.44^{\mathrm{c}}$ & $16.89 \pm 1.62^{\mathrm{a}}$ & $\leq 0.001 *$ \\
\hline & & $\mathrm{C} 2$ & $2.97 \pm 0.54^{b}$ & $1.13 \pm 0.36^{\mathrm{c}}$ & $23.09 \pm 0.80^{\mathrm{a}}$ & $\leq 0.001 *$ \\
\hline & \multirow{2}{*}{ A3 } & C1 & $3.00 \pm 0.74^{\mathrm{b}}$ & $1.44 \pm 0.44^{\mathrm{c}}$ & $16.69 \pm 1.21^{\mathrm{a}}$ & $\leq 0.001^{*}$ \\
\hline & & $\mathrm{C2}$ & $2.93 \pm 0.72^{b}$ & $1.03 \pm 0.31^{\mathrm{c}}$ & $23.46 \pm 0.77^{\mathrm{a}}$ & $\leq 0.001 *$ \\
\hline
\end{tabular}

Different letter within each row indicates significant difference. 


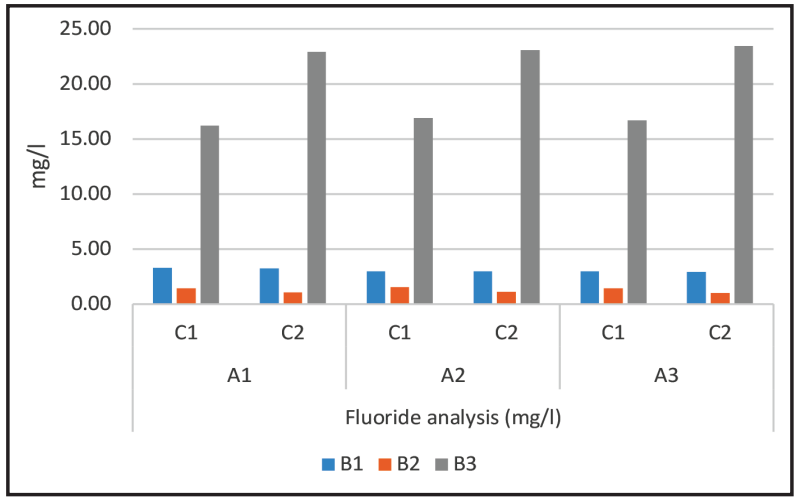

Figure (2) Bar chart showing mean fluoride analysis (mg/l) of different immersion media with the other variables.

\section{DISCUSSION}

The usage of nanoparticles was represented as a considerable issue of dental researches. Nanoparticles are openly used as strengthens fillers in dental restorations, since they have a noticeable effect on their performance when integrated in small amounts in comparison with micro fillers. Nanoparticles are capable of penetration into the tooth micro-pores due to their small size, improving the properties of the bond layer ${ }^{(18)}$. So that, glass ionomer restorations modified with montmorillonite nanoparticles were selected for investigation in this study.

Glass ionomer cements (GIC) are dependent on the interaction between polyacrylic acid and silicate glass powder, so in treatment of erosion and caries found in low stress bearing areas they are predominately has a beneficial effect. So that, class V cavities were done for the in vitro study. Standardized trapezoidal class $\mathrm{V}$ cavities were chosen to be prepared in the gingival one third of the labial surface of teeth with the preparation. The bur was replaced after every fifth preparation to ensure cutting efficiency. Each cavity was measured with a William's graduated probe to ensure uniform size. after 24 hours of restoration of the cavities, the excess restorative material was removed using a diamond stone with small grid at high speed to ensure complete setting of the material and so the cavities exhibited smooth surfaces to be ready for microhardness test. After finishing of the restorations, the samples maturation was done in an incubator for 1 hour at $37^{\circ} \mathrm{C}$ to mimic oral condition ${ }^{(14)}$.

Many inventors have proposed that the in vitro fluoride analysis of dental materials has been made in situations which do not resemble development of caries and fluoride effect. Distilled water, which does not represent the oral fluid, has been the medium frequently used to evaluate fluoride release. Some studies have used artificial saliva, although this medium is more appropriate, it does not simulate the caries process. In addition when the fluoride released from restorative materials was compared using Distilled water or artificial saliva, reversal results were found. Considering that dental caries depends on the equilibrium between demineralization and remineralization during the $\mathrm{pH}$-cycling into dental plaque, simulate these conditions ${ }^{(19)}$. Therefore, the intent of this study was to measure the fluoride release from glass-ionomer restorations in conventional solutions i.e. distilled water (as a negative control) and artificial saliva (positive control) and in a pH-cycling model (demineralizing and remineralizing solutions) to simulate oral condition and the dynamic situation. The cola beverage was selected as a demineralizing solution due to its low calcium and fluoride ions other than the low $\mathrm{PH}^{(20)}$. The solution was changed every day for ensuring that the undersaturation status kept constant, and to stop a build-up of fluoride within the immersion solutions which contain the specimens.

Stability of the acid-base reaction of glass ionomer cements is mandatory, so the one-week storage period was chosen for as the highest alteration in hardness has been occurred through the first week. One month storage period was chosen allowing long contact period for evaluation of the effect of prolonged contact with acidic beverage ${ }^{(12)}$. Also the in vitro evaluation over 15 days represents an in vivo situation of high caries challenge ${ }^{(19)}$. 
Hardness is a significant surface feature which affects the scratching and polishing appearance of the material and determines its durability after its subjecting to indentation and penetration ${ }^{(21)}$. Hardness testing procedure (Microhardness) was applied when the samples are relatively thin or small. The microhardness of materials can be computed in many ways. Vickers microindentation test is considered one of the famous ways of hardness testing. The material is enforced by 136-degree diamond pyramid-shaped indenter with a specified capacity implementation. The Vickers indenter always makes a geometrically identical indentation at all test loads. The tone created by the Vickers indenter is more obvious when compared to the tone of another indenters ${ }^{(16)}$.

The theory of action of ion chromatography (Dionex ICS-5000) which measures fluoride release, depends on physico-chemical separation methods having the allocation of an ingredient between a mobile phase and a stationary phase. The stationary phases contain a polystyrene resin copolymerized with divinylbenzene and modified with ion-exchange set. A buffered hydrous solution recognized as the mobile phase transfer the specimens from the loop onto a column that holds some form of stationary phase material ${ }^{(9)}$.

According to the results of the study, the Dellite 43 modified glass ionomer showed the highest values of microhardness between the tested materials followed by Dellite LVF modified glass ionomer and finally conventional glass ionomer. These results were the same after immersion in different solutions for different periods. There were statistically significant difference between the mean microhardness of the tested materials.

These results may be attributed to that, the organoclay was dissolved in the liquid part of promedica watery solution (polyacrylic acid PAA). This integration increased the space among layers of the nanoclay for trapping of organic molecules. Ion exchange with sodium ions on silicate plates and the reaction of PAA with organoclay appears through the operation of nanoclay incorporation. Through formation of hydrogen bonds or by iondipole interaction, polymer chains of PAA can adsorb onto the face of clay architecture. Ionic bonding at the interface between GIC polymer matrix and the nanoclay was obtained by various interactions of nanoclay either chemical or physical resulting in an improving of its mechanical properties ${ }^{(11)}$. Increasing of space between layers (d-spacing) registered for organo-modified montmorllonite (MMT) revealed that, the clay had stretched layers which provides raising the chance of its penetration by the polyacrylic acid chains of the GIC restoration. It was assumed that, the organomodified MMT had a strengthen action on the conventional GICs due to increased d-spacing ${ }^{(22)}$.

The structure of polyacid and glass powder affect the mechanical properties of GICs. GICs reinforcement can be modified by incorporation of nanoclay plates in the polymeric matrix. Choosing appropriate nanoclay for PAA is essential for the development of nanoclay-modified glass ionomer dental restoration. A regular incorporation of nanoparticles with PAA matrix is desired for enhancement of characteristics of the set material. Increasing of the interlayer d-spacing of the nanoclay was created by the dispersion of nanoclays in hydrous solutions of $\mathrm{PAA}^{(11)}$. Also the integration of nanoclay in a polymer matrix enhances the mechanical properties, particularly the elastic modulus of systems when the particles have suitable allocation and dispersion leading to a higher earn in surface area of these particles increasing the strengthening effect ${ }^{(18)}$.

However, the feedback of the study was in contrast with some studies ${ }^{(23,24)}$. The incorporation of Ca-MMT clay to the GI restoration leads to noticeable decrease in the in vitro wear resistance. Addition of 12-amino-dodecanoic acid treated (ADA-MMT) organoclay to conventional GIC at $0.5-2.5 \mathrm{wt} \%$, leads to a noticeable improvement in the mean compressive fracture strength, while addition of $\mathrm{Ca}-\mathrm{MMT}$ clay to conventional GIC, leads to a respectable decrease the in mean strength. 
In this study, when specimens were subjected to challenge of erosion, the microhardness of restorative materials showed statistically significant lower value than that showed for the control specimens placed in distilled water (negative control) and artificial saliva (positive control), so there was no statistically significant difference between the mean microhardness of specimens which were sunken in the distilled water and artificial saliva but there was a significant difference between the first two solution and the demineralizing - remineralizing solutions ( $\mathrm{pH}$ cycling). This may attributed to the acid impedance of conventional GIC which is relatively low. The fluoro-alumino-silicate glasses are degraded after the acid attack, releasing fluoride, calcium and aluminum ions leading to reduction in surface hardness. The matrix degradation of glass particles of GIC, may affected by disintegration of the siliceous hydrogel layer ${ }^{(12)}$.

Releasing of fluoride depends on $\mathrm{pH}$; a great level of fluoride is released from restorative materials when PH of the solution is low. Increase rate of fluoride release supposes an increase material dissolution and can recorded on the surface characteristics and hardness rates. This may be proven, as a statistically significant relation is noticed between hardness and release of fluoride for many materials ${ }^{(10)}$. Subjecting of restorative materials in the acidic medium leads to reduction of the surface hardness and causing roughness in the surface which increased by time, after monitoring with SEM photomicrographs $^{(25)}$. PH cycling process causing sever reduction in the microhardness of different glass ionomer restorations ${ }^{(26)}$.

The mean fluoride release of Dellite 43 modified glass ionomer was higher than mean fluoride release of Dellite LVF modified glass ionomer and the mean fluoride analysis of both were higher than of conventional glass ionomer regarding to the results of this study. This is may be caused by the large surface area created by interlayer d-spacing recorded by Dellite 43 modified glass ionomer that increases the acid base reaction and has the ability to release fluoride from the powder rapidly, raising the fluoride release rate of the material but there was no statistically significant difference between the mean fluoride release of the tested materials with different immersion solutions and periods.

The mean fluoride release of materials which sunken in distilled water was higher than mean fluoride release of them when they were kept in artificial saliva and the mean fluoride release of both were much lower than of demineralizing - remineralizing solutions ( $\mathrm{pH}$ cycling). The results revealed that, there was a statistically significant difference between the mean fluoride releases of the materials with the different solutions. This may be due to that, distilled water does not seem to be the most appropriate medium to compare the fluoride release from dental materials, it has neutral environment with no changes in $\mathrm{pH}$ so it was with no effect on fluoride release.

Artificial saliva was selected as a second solution for fluoride releasing to resemble the natural oral environmental status, although, repeating the same properties of human saliva is unattainable because of the unstable ease of natural saliva. The lowest values reported in the second solution, due to the presence of cations and anions in artificial saliva and an ionic action on the disintegration and the release of fluoride ions may be enhanced by calcium ions in the testing medium, due to $\mathrm{CaF} 2$ formation ${ }^{(27)}$. PH cycling model was used to represent a dynamic status, because dental caries acts as a process of alternating demineralization and remineralization phenomena that were a direct function of conditions that maintain a critical $\mathrm{pH}$ in the mouth. Although artificial saliva represents the oral environment, $\mathrm{pH}$-cycling solutions would be preferable to investigate the anticariogenic possibility of dental materials, as they reproduce the dynamic status of caries. The raising level of fluoride in acidic medium could be discussed by the reality that, low $\mathrm{pH}$ leads to rises the disintegration of the material causing a high fluoride value in the acidic solution. 


\section{CONCLUSION}

Under the conditions of the present study, the conclusion were that the proposed $\mathrm{pH}$ cycling model affects the microhardness of the tested materials while improves their fluoride release. Although the incorporation of montmorillonite into glass ionomer liquid improves the microhardness of the materials especially the organo- modified type, it has yet, no effect on its fluoride release regardless of the different immersion solutions or time of immersion.

\section{REFERENCES}

1. Ilie N, Hickel R, Valceanu AS, Huth KC. Fracture toughness of dental restorative materials. Clin Oral Investig. 2012; 16:489-98.

2. Moshaverinia A, Roohpour N, Chee WW, Schricker SR. A review of polyelectrolyte modifications in conventional glass-ionomer dental cements. J Mater Chem B. 2012; 22: 2824-33.

3. Davidson C. Advances in glass-ionomer cements. JMID. $2009 ; 2: 3-15$

4. Okada A, Usuki A. Twenty years of polymer-clay nanocomposites. Macromol Mater Eng. 2006; 291:1449-76.

5. Fareed MA, Stamboulis A. Nanoclays reinforced glass ionomer cements: dispersion and interaction of polymer grade (PG) montmorillonite with poly (acrylic acid). J. Mater. Sci.: Mater. Med. 2014; 25:91-9.

6. Solhi L, Atai M, Nodehi A, Imani M, Ghaemi A, Khosravi K. Poly (acrylic acid) grafted montmorillonite as novel fillers for dental adhesives: synthesis, characterization and properties of the adhesive. Dent Mater. 2012; 28:369-77.

7. Bhattacharya A, Vaidya S, Tomer AK, Mangat P, Raina AA. Evaluation and comparison of physical properties and fluoride release of newly introduced ceramic reinforced glass-ionomer restorative material with other glass ionomer cements-An in vitro study. Int. J Appl Dent Sci 2017; 3: 486-92.

8. deMoraes MD, de Melo MA, Bezerra DD, Costa LS, Saboía VD, Rodrigues LK. Clinical study of the cariespreventive effect of resin-modified glass ionomer restorations: aging versus the influence of fluoride dentifrice. $\mathrm{J}$ Investig Clin Dent. 2016; 7 180-6.

9. Elgamily H, Ghallab O, El-Sayed H, Nasr M. Antibacterial potency and fluoride release of a glass ionomer restorative material containing different concentrations of natural and chemical products: An in-vitro comparative study. J Clin Exp Dent. 2018; 10:312-20.

10. Silva KG, Pedrini D, Delbem AC, Cannon M. Effect of $\mathrm{pH}$ variations in a cycling model on the properties of restorative materials. Oper Dent. 2007; 32:328-35.

11. Fareed MA, Stamboulis A. Effect of nanoclay dispersion on the properties of a commercial glass ionomer cement. Int J Biomater. 2014; 1-10

12. Francisconi LF, Honório HM, Rios D, Magalhães AC, Machado MD, Buzalaf MA. Effect of erosive $\mathrm{pH}$ cycling on different restorative materials and on enamel restored with these materials. Oper Dent. 2008; 33:203-8.

13. Prabhakar AR, Jibi Paul M, Basappa N. Comparative evaluation of the remineralizing effects and surface micro hardness of glass ionomer cements containing bioactive glass (S53P4): an in vitro study. Int J Clin Pediatr Dent. 2010; 3:69-77

14. Fatima N, Abidi SY, Jat SA. Effect of different tetra pack juices on microhardness of direct tooth colored-restorative materials. Saudi Dent. J.2013; 25:29-32.

15. Hicks MJ, Flaitz CM. Enamel caries formation and lesion progression with a fluoride dentifrice and a calcium-phosphate containing fluoride dentifrice: a polarized light microscopic study. ASDC J Dent Child. 2000; 67:21-8.

16. Borzabadi-Farahani A, Lynch E. Influence of thermo-light curing with dental light-curing units on the microhardness of glass-ionomer cements. Int J Periodontics Restorative Dent. 2016; 36:425-30.

17. ISO I. 6507-1: 2018. Metallic Materials-Vickers Hardness Test-Test Method.

18. Menezes LR, Silva EO. The use of montmorillonite clays as reinforcing fillers for dental adhesives. Mater. Res. 2016; 19:236-42.

19. Carvalho AS, Cury JA. Fluoride release from some dental materials in different solutions. Oper Dent. 1999; 24:14-9.

20. Xavier AM, Sunny SM, Rai K, Hegde AM. Repeated exposure of acidic beverages on esthetic restorative materials: An in-vitro surface microhardness study. J Clin Exp Dent. 2016; 8:312-7.

21. Wang L, D'Alpino PH, Lopes LG, Pereira JC. Mechanical properties of dental restorative materials: relative contribution of laboratory tests. J Appl Oral Sci. 2003; 11:162-7.

22. Dowling AH, Stamboulis A, Fleming GJ. The influence of montmorillonite clay reinforcement on the performance of a glass ionomer restorative. J. Dent. 2006; 34:802-10. 
23. Dowling AH, Fleming GJ. The impact of montmorillonite clay addition on the in vitro wear resistance of a glassionomer restorative. Dent. 2007; 35:309-17.

24. Sajjad A, Bakar WZ, Mohamad D, Kannan TP. Various recent reinforcement phase incorporations and modifications in glass ionomer powder compositions: A comprehensive review. J Int Oral Health. 2018; 10:161-7.

25. Hengtrakool C, Kukiattrakoon B, Kedjarune-Leggat U. Effect of naturally acidic agents on microhardness and surface micromorphology of restorative materials. Eur. J. Dent. 2011; 5:89-100.

26. Kaki GD. The effects of $\mathrm{pH}$ changes on the microhardness of three fluoride releasing restorative materials: An in vitro study. EC Dent Sci. 2018; 17:1645-51.

27. Alam MK. Fluoride release from dental restorations in de-ionized Water and artificial saliva. Int Med J. 2013; 20:635-8. 